\title{
Space-Time Coding for UMTS. Performance Evaluation in Combination with Convolutional and Turbo Coding
}

\author{
Roger Gaspa, Javier R. Fonollosa \\ Department of Signal Theory and Communications \\ Universitat Politècnica de Catalunya \\ c/ Jordi Girona, 1-3, Mòdul D-5, Campus Nord UPC \\ 08034 Barcelona, SPAIN \\ e-mail: \{rgaspa, fono\}@gps.tsc.upc.es
}

\begin{abstract}
Space-time codes provide both diversity and coding gain when using multiple transmit antennas to increase spectral efficiency over wireless communications systems. Space-time block codes have already been included in the standardization process of UMTS in conjunction with conventional channel codes (convolutional and turbo codes). We discuss different encoding and decoding strategies when transmit diversity is combined with conventional channel codes, and present simulations results for the TDD and FDD modes of UTRA.
\end{abstract}

\section{Introduction}

Employment of multi-element antenna arrays at both transmit and receive sites is capable of enormous theoretical capacity over wireless communications systems. Time-varying fading due to multipath propagation can be effectively combated by using more than one transmit antennas, providing transmit diversity advantage. Different techniques to achieve transmit diversity have been reported in the literature. Recently, space-time codes have been formulated form a coding perspective to provide both coding and diversity gain. Space-time codes may be based on a trellis structure (Space-Time Trellis Codes [1]) or be block-oriented (Space-Time Block Codes [2]). On one hand, STTC provide transmit diversity while they can also achieve coding gain since long memory codes can be used at the encoder. On the other hand, STBC act in a block-wise manner so that only those bits (symbols) being encoded

\footnotetext{
* This work was partially supported by the European Commission under Project IST-1999-11729 METRA; the Spanish Government (CICYT) TIC98-0412, TIC98-0703, TIC99-0849; and the Catalan Government (CIRIT) 1998SGR 00081
}

within a block will be 'connected' thanks to the code constrains, hence providing small coding gain but a very simple decoding algorithm. In [3,4,5] Foschini et al. presented a high efficiency transmit diversity scheme, namely BLAST (Bell Labs Layered Space-Time Architecture), which basically demultiplexes the data stream to different substreams, each one sent to each antenna. Finally, these substreams, which might have been independently encoded by a channel code, are modulated and sent from their respective antenna.

In general, we have to consider a system concatenating an outer channel code with a space-time code (inner code) as in 3rth generation mobile systems, where STBC using two transmit antennas (Alamouti code [alamouti]) have already been included in the standardization process of UMTS in conjunction with conventional channel codes (convolutional and turbo codes) $[6,7]$.

In this paper we present performance results of this concatenated scheme for the TDD and FDD modes of UTRA, as well as a comparison with other transmit diversity schemes. Different decoding approaches are analyzed in terms of probability of error and decoding complexity.

The paper is organized as follows. Sec.II. describes the system model and the encoder and decoder structure. Sec.III. presents different transmit schemes and their decoding algorithms. Simulation results are analyzed in Sec.IV. Finally, brief conclusions are given in Sec.V.

\section{System Model}

Figure 1 shows the transmit scheme of the system considered in this paper. Information bits $b_{i}$ are first encoded by an outer channel code, which can be a convolutional code or a turbo code as specified by 3 GPP. The packet length to be encoded (N) depends on the information bit rate and TTI (Transmission Time Interval) but block segmentation should be performed if it is larger than the maximum admissible coding length, i.e. $\mathrm{N}=504$ 
and $\mathrm{N}=5114$ for convolutional codes and turbo codes, respectively. The frame for the UMTS $3^{\text {th }}$ generation mobile system is divided into 15 timeslots, each one of 2560 chips at chip rate of $3.84 \mathrm{Mchip} / \mathrm{s}$, hence each frame is transmitted in $10 \mathrm{~ms}$. TTI takes values of $10,20,40$ or $80 \mathrm{~ms}$ depending on the service constraints.

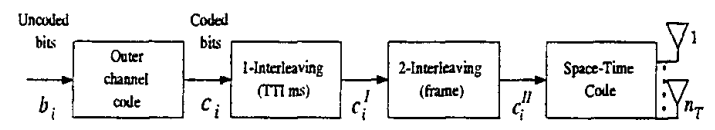

Figure 1: Block diagram of the transmitter.

Coded bits $c_{i}$ are fed to a first block interleaver which interleaves across different frames if TTI is larger than $10 \mathrm{~ms}$. Radio frame segmentation is performed before coded bits are interleaved (only within a frame) by a second block interleaver. Both interleaver patterns are specified in $[6,7]$. Finally, the space-time coder outputs $d_{i}(t) i=1 . . n_{T}$ symbol constelation points at each time slot $\mathrm{t}$, which are transmitted simultaneously from each antenna $i$, $i=1 . . n_{T}$.

The signal constellation is scaled such that the total transmitted energy in normalized to unity. We assume that the energy is equally distributed along all transmit antennas, hence the average energy out of each antenna is $1 / n_{T}$. We consider a system employing $n_{T}=2$ transmit antennas and $n_{R}=2$ receive antennas. We restrict ourselves to $n_{T}=2$ since only two transmit antennas are being considered by the 3 GPP standardization group, and to a QPSK constellation.

The received signal is a noisy filtered superposition of the transmitted signals,

$$
r_{j}(t)=\sum_{i=1}^{n_{Y}} h_{i, j}(t) d_{i}(t)+n_{j}(t)
$$

where $r_{j}(t) j=1 . . n_{R}$ denotes the received signal at time $\mathrm{t}$ at receive antenna $j, j=1 . . n_{R}$, and $n_{j}(t)$ represents an additive white Gaussian noise modeled as independent samples of a zero mean complex Gaussian random variable with variance $\mathrm{N}_{0} / 2$ per dimension . $h_{i, j}(t)$ denotes the fading coefficient from transmit antenna $i$ to receive antenna $j$ at time $t$. Since the angular spread seen at the mobile is in general relatively large due to local scattering we assume that path gains between antennas at the mobile are independent. However this assumption is not applicable at the base station, where antennas are correlated owing to a small angular spread.
Figure 2 shows the receive path of the system being considered. The space-time decoder computes the soft(bit)-values (Log-Likelihood) of the transmitted bits from the received signal $r_{j}(t), j=1 \ldots n_{R}$ and channel estimates $\bar{h},$. In this paper we consider ideal channel estimation, that is, $\bar{h}=h$. After de-interleaving, the estimated softvalues are fed to the outer channel decoder.

As mentioned above, we can consider this system as the concatenation of an inner code with an outer code, hence it is an open question whether an iterative procedure that feeds back the output of the outer code to the inner code will improve the overall system performance, as it happens with turbo decoding. This feedback information has to be interleaved by the same interleaving patters that where present in the transmitter site. This iterative procedure, as well as the decoding algorithm for the space-time code, are described for each transmit scheme under consideration in next section.

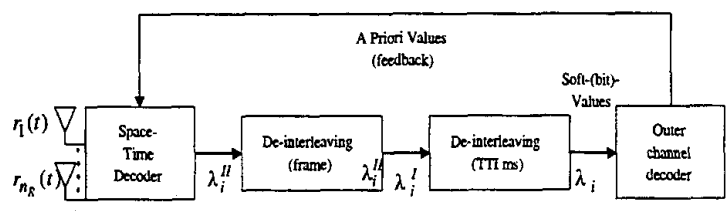

Figure 2: Block diagram of the receiver

The turbo code (outer code) is decoded using the iterative decoding algorithm given in [9]. The decoder, when convolutional codes are used as outer codes, is simply the Viterbi algorithm if only hard-decisions are required at the output, but the SOVA [10], log-MAP or max-log-MAP [11] algorithms are used to compute softvalues for the decoded bits if needed.

\section{Transmit Diversity Schemes}

\section{III.1 STBC: Space Time Block Codes}

Space-time block codes have been described in [1] as a generalization of the Alamouti code to more than 2 transmit antennas. Figure 3 depicts a general space-time block encoder, $K^{*} m$ coded bits $c_{i}$ from the outer channel code are mapped to the appropriate signal constellation, i.e. QPSK, to form $K$ complex symbols $s_{i}, i=1 . . K$ (where $m$ represents the number of bits per symbol, i.e. $2^{m}$ constellation points). The STBC encoder maps the $K$ input symbols into a $p \cdot n_{T}$ matrix $G$, the columns representing those symbols transmitted from each transmit antenna 
during $p$ different time slots. Therefore the rate of this code is $k p$. In particular, the Alamouti code maps two consecutive symbols to a $2 \times 2$ space-time matrix defined by $(K=2, p=2)$

$$
G_{A}=\left(\begin{array}{cc}
s_{1} & s_{2} \\
-s_{2}^{*} & s_{1}^{*}
\end{array}\right)
$$

where ${ }^{*}$ stands for complex conjugate. Note that this code has code rate $=1$, hence it does not require any bandwidth expansion.

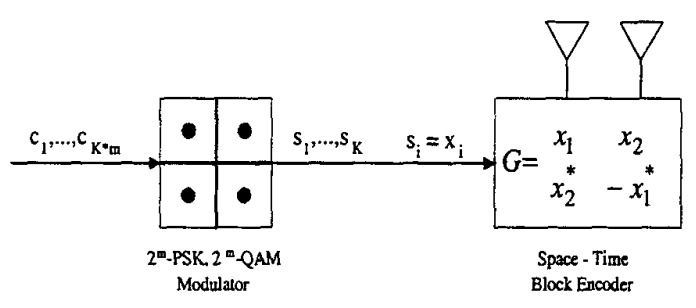

Figure 3: Block diagram of a Space-Time Block Encoder.

The received signal at each receive antenna feeds the STBC decoder which should provide soft-(bit)-values to the outer channel decoder. If Alamouti code is used, soft(symbol)-values for symbols $s_{l}$ and $s_{2}$ can be easily obtained:

$$
\begin{aligned}
& \sum_{j=1}^{n_{R}} r_{j}(\mathrm{I}) h_{1 j}{ }^{*}+r_{j}(2)^{*} h_{2 j}=\sum_{j=1}^{n_{R}}\left(\left|h_{1 j}\right|^{2}+\left|h_{2 j}\right|^{2}\right)_{1}+n_{j}(\mathrm{I}) h_{1 j}{ }^{*}+n_{j}(2)^{*} h_{2 j} \\
& \sum_{j=1}^{n_{R}} r_{j}(\mathrm{I}) h_{2 j}{ }^{*}-r_{j}(2)^{*} h_{1, j}=\sum_{j=1}^{n_{R}}\left(\left|h_{1 j}\right|^{2}+\left|h_{2 j}\right|^{2}\right)_{2}+n_{j}(\mathrm{I}) h_{2 j}{ }^{*}+n_{j}(2)^{*} h_{1 j}
\end{aligned}
$$

assuming that path gains $h_{i, j}$ do not change during the transmission of $s_{1}$ and $s_{2}$. We have to calculate soft-(bit)values from the computed soft-(symbol)-values before outer channel decoding. For a QPSK that maps bits $\left\{b_{0}, b_{l}\right\}$ to symbol constellation $s=b_{0}+j b_{l}$, the soft-(bit)values are easily computed as:

$$
\begin{aligned}
& \lambda_{b_{0}}=\operatorname{Real}\{s\} \\
& \lambda_{b_{1}}=\operatorname{Imag}\{s\}
\end{aligned}
$$

Since the Alamouti code provides diversity gain but no coding gain, we can not expect any improvement trough iterative decoding and, consequently, a feed-forward decoding scheme without feedback information was implemented.

\section{III.2 STTC: Space Time Trellis Codes}

STTC [2] are defined over a trellis structure where each input symbol has associated $n_{T}$ symbols, each one of them transmitted from each antenna. The STTC simulated in this paper are those proposed in [2]. We note again that no bandwidth expansion is required for this codes. The decoding step is based on maximum-likelihood sequence estimation (MLSE).

If the target is to output hard decisions from the decoder, a simply Viterbi algorithm can be used to compute the path with the lowest accumulated metric. The branch metric from state $s$ to $s^{\prime}$ is given by,

$$
\sum_{j=1}^{n_{R}}\left|r_{j}(t)-\sum_{i=1}^{n_{T}} h_{i, j} q_{i}(t)\right|^{2}
$$

where $q_{1}, q_{2}, \ldots q_{n T}$ are the symbols associated to the transition from state $s$ to $s^{\text {. }}$

However, it is also possible to compute soft-values by using any MAP-based algorithm (i.e. MAP, log-MAP, max-log-MAP [11,12]). An iterative procedure could be performed in order to improve the performance of the overall resulting system.

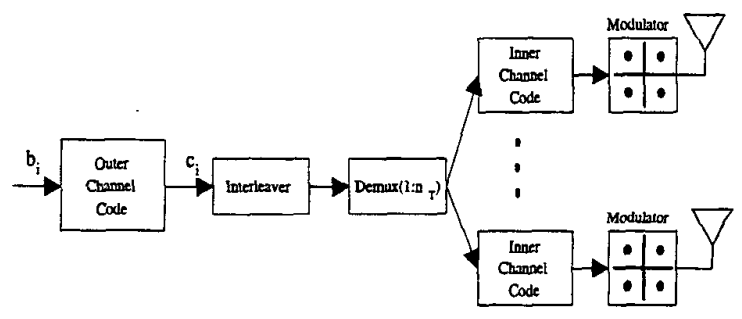

Figure 4: Block diagram of a transmitter using more than one transmit antenna.

Nevertheless, we note that both codes are serially concatenated, which implies that the outer code should calculate soft-values for both coded and uncoded bits, that is to output soft-values not only for those bits corresponding to the input to the outer channel encoder but also for those bits actually encoded by the channel encoder. However, a MAP algorithm to compute softvalues for coded bits requires higher computational complexity than the 'conventional' MAP algorithm. Hence, in order to keep the decoding complexity as low as possible, we only feed-back 'a priori' values for those 
uncoded bits out of the outer code, whereas the 'a priori' values for coded bits are set to zero.

\section{III.3 Transmit Diversity schemes without 'space coding'}

STBC generate orthogonality between transmit channels so that the receiver can separate those symbols transmitted from each antenna. STTC are designed according to the rank and determinant [2] criteria to maximize the diversity and coding gain, respectively.

Of course, one could transmit different symbols from different antenna without 'space' encoding the signal to provide orthogonality.

Different publications appeared in the literature using several transmit antennas without using STBC nor STTC. Layered space-time codes (i.e. BLAST) developed by Foshicni et al. [13] or a Turbo Coded Modulation with antenna diversity [14] are examples of those publications.

BLAST is a bandwidth-efficient transmitter architecture, which takes advantage of the spatial dimension by transmitting and detecting a number of independent co-channel data streams (substreams) each one transmitted from different antenna $[3,4,5]$.

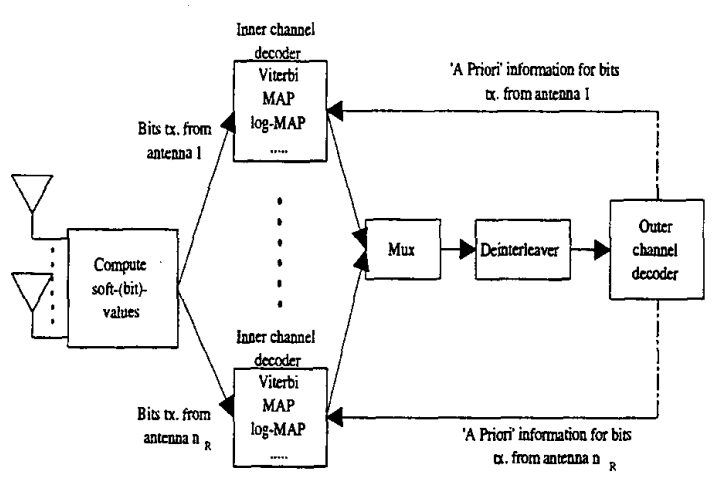

Figure 5: Block diagram of the receiver with feedback information.

Basically, the bitstream (coded or uncoded data from previous blocks) is demultiplexed into different substreams (one per each transmit antenna), encoded into symbols and fed to its respective transmit antenna. On one hand, inter-substream coding, such that some redundancy between substreams is incorporated, leads to D-BLAST. On the other hand V-BLAST does not encode the signal (or, at least, only individual substream coding is applied).

In general, we could set up a transmit diversity system as the one presented in Figure 4. Bits are first encoded by an outer channel code. After demultiplexing the resulting coded bits, another channel code is applied to each substream and finally mapped into the appropriate signal constellation. Again, the receiver structure could incorporate feedback mechanisms to iteratively decode the transmitted bit-stream, as shown in Figure 5.

As described in [3] the detection process for layered space-time coding of all substreams is divided into three key aspects: interference nulling, interference canceling and compensation. Interference nulling projects out interference from those substreams not yet detected, interference canceling substracts out interference of those substreams already detected, finally stronger substreams compensates weaker ones. Alternatively, we can compute the log-likelihood ratios of the transmitted bits directly for the received signals $r_{j}(t)$. Suppose that symbols $d=\left\{d_{l}(t)\right.$, $\left.\ldots, d_{n T}(t)\right\}$ have been transmitted at time instant $t$. For clarity we will drop the time reference hereafter, i.e. $\boldsymbol{d}=\left\{d_{1}, \ldots, d_{n T}\right\}$. Let us denote by $\boldsymbol{b}=\left\{b_{1}, b_{2}, \ldots, b_{m}, \ldots\right.$, $\left.d_{m^{*} n T}\right\}$ the sequence of bits that correspond to $d=\left\{d_{i}, \ldots\right.$, $\left.d_{n T}\right\}$, where $m$ represents the number of bits per symbol. The log-likelihood for bit $b_{i}$ is given by,

$$
\lambda_{b_{i}}=\log \frac{\operatorname{Pr}\left(b_{i}=1 \mid r_{1}, r_{2} . ., r_{n_{R}}\right)}{\operatorname{Pr}\left(b_{i}=0 \mid r_{1}, r_{2} \ldots, r_{n_{R}}\right)}
$$

Assuming that all symbol constellation points are equally likely and that the received signals at each receive antenna are conditionally independent,

$$
\operatorname{Pr}\left(r_{1}, r_{2}, \ldots, r_{n_{R}} \mid \mathbf{d}\right)=\prod_{j=1}^{n_{R}} \operatorname{Pr}\left(r_{j} \mid \mathbf{d}\right)
$$

which leads to [14],

$$
\lambda_{b_{i}}=\log \frac{\sum_{\substack{\text { d:d }=\text { mapping(b) } \\ b_{i}=1}} \prod_{j=1}^{n_{R}} \exp \left(-\frac{\left|r_{j}-\sum_{i=1}^{n_{T}} h_{i, j} d_{i}\right|^{2}}{N_{0}}\right)}{\sum_{\substack{\text { d:d d mapping(b) } \\ b_{i}=0}} \prod_{j=1}^{n_{R}} \exp \left(-\frac{\left|r_{j}-\sum_{i=1}^{n_{T}} h_{i, j} d_{i}\right|^{2}}{N_{0}}\right)}
$$

where the mapping function maps bit sequence $b$ to symbol sequence $d$.

Again, BLAST architecture using channel codes with code rate $1 / n_{\mathrm{T}}$ does not require any bandwidth expansion, and thus can be compared fairly with the STTC and STBC. 


\section{Simulation Results}

Simulations have been carried out to analyze the performance of the aforementioned transmit diversity systems in tems of Bit Error Rate (BER) and Block Error Rate. A block error occurs if at least one bit of the burst is in error. Several simulations have been run for different concatenation schemes, services and user speed, which are detailed in Table 1. According to 3GPP specifications, block segmentation occurs if the uncoded packet length is larger than the maximum allowed encoding size. The input block is divided into different packets and they are encoded separately.

The outer channel code used either a convolutional code or a turbo code. The convolutional code is a feedforward convolutional code with rate $1 / 3$ and constraint length $K_{c c}=9$ whose polynomial generators are $\{557,663$, 771 \}, expressed in octal form.

The turbo code is constructed by parallel concatenation of two recursive 8-state convolutional codes. The turbo code rate is $1 / 3$ with transfer function,

$$
G(D)=\left[1, \frac{1+D^{2}+D^{3}}{1+D+D^{3}}\right]
$$

The turbo code internal interleaver, as well as the interframe and intra-frame interleavers, are those defined in $[6,7]$.

The following transmit diversity schemes have been considered: STBC (Alamouti Code), STTC (4-states), and different configurations for the structure presented in Figure 4, that is:

TD.1) Outer channel code: turbo code rate $1 / 3$. Inner channel code: 8 -state convolutional code rate $1 / 2$.

TD.2) Outer channel code: turbo code rate $1 / 6$. Non inner channel code.

Note that both schemes do not require any bandwidth expansion compared to a turbo code rate $1 / 3$, since there are 2 transmit antennas. We assume that the system is using two transmit and two receive antennas.

Figure 6 and Figure 7 show the performance of the complete coding scheme for a data rate of $4.1 \mathrm{kbps}$ of STBC and 4-states STTC concatenated with a convolutional code $(\mathrm{CC})$, at vehicle speed of $3 \mathrm{kmh}$ and $60 \mathrm{kmh}$, rspectively. The packet length is 164 bits (with 16 CRC bits). The STBC improve the STTC performance by $1 \mathrm{~dB}$ at same BER. At $60 \mathrm{kmh}$ the interleaver obtains an additional gain since the channel changes during the transmission interval. Note that in this case for the TD.1 scheme the outer channel code is the convolutional code defined by 3 GPP. No significant iteration gain is obtained using feedback information from the inner to the outer channel code.

\begin{tabular}{|c|c|c|c|c|}
\cline { 2 - 5 } \multicolumn{1}{c|}{} & $\begin{array}{c}\mathbf{4 . 1} \\
\text { Kbps }\end{array}$ & $\begin{array}{c}\mathbf{6 4} \\
\text { Kbps }\end{array}$ & $\begin{array}{c}\mathbf{3 8 4} \\
\text { Kbps }\end{array}$ & $\begin{array}{c}\mathbf{2} \\
\text { Mbps }\end{array}$ \\
\hline $\begin{array}{c}\text { Uncoded } \\
\text { packet }\end{array}$ & 164 & 1280 & 7680 & 80000 \\
bits & bits & bits & bits \\
\hline $\begin{array}{c}\text { CRC } \\
\text { per bock }\end{array}$ & 16 & 16 & 16 & 16 \\
bits & bits & bits & bits \\
\hline $\begin{array}{c}\text { TTI } \\
\text { (ms) }\end{array}$ & 40 & 20 & 20 & 40 \\
\hline $\begin{array}{c}\text { Block } \\
\text { segmenta } \\
\text { tion }\end{array}$ & 1 & 1 & 2 & 16 \\
\hdashline $\begin{array}{c}\text { Outer } \\
\text { channel } \\
\text { code }\end{array}$ & $\begin{array}{c}\text { Conv. } \\
\text { code }\end{array}$ & $\begin{array}{c}\text { Turbo } \\
\text { code }\end{array}$ & $\begin{array}{c}\text { Turbo } \\
\text { code }\end{array}$ & $\begin{array}{c}\text { Turbo } \\
\text { code }\end{array}$ \\
\hline
\end{tabular}

Table 1: Data rates and paramters assocoated used in simulations.

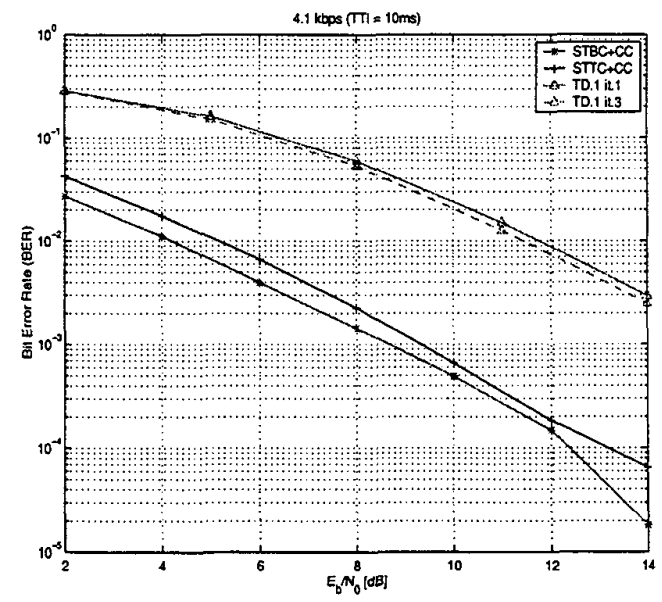

Figure 6: BER for $4.1 \mathrm{Kbps}$ service at $3 \mathrm{kmh}$.

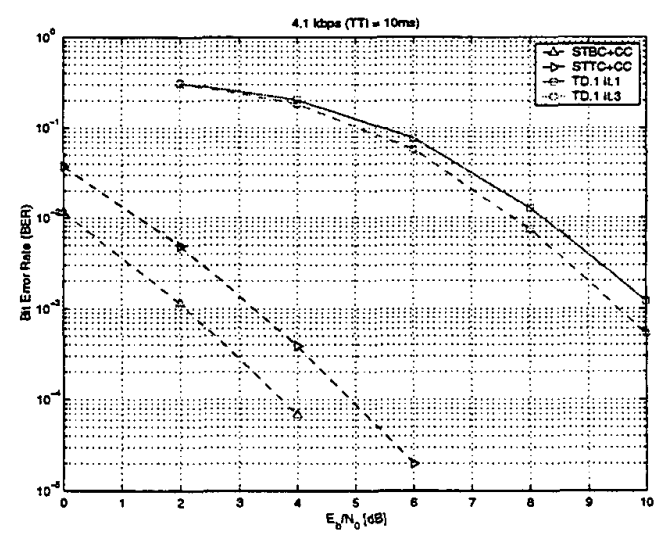

Figure 7: BER for $4.1 \mathrm{Kbps}$ service at $60 \mathrm{kmh}$. 


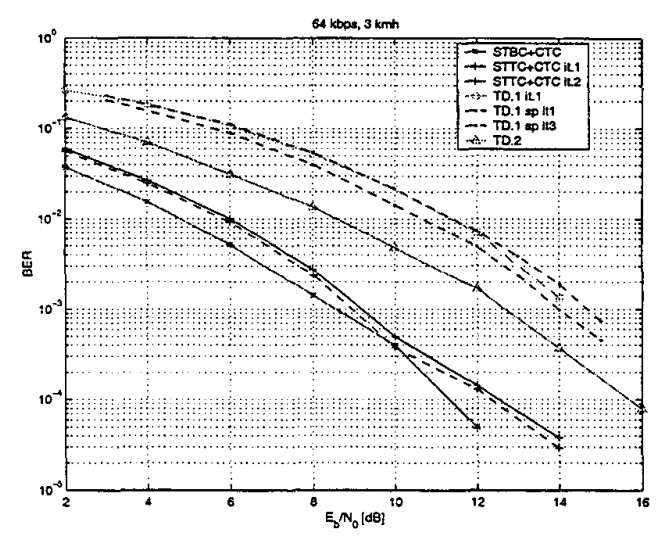

Figure 8: BER for $64 \mathrm{Kbps}$ service at $3 \mathrm{kmh}$

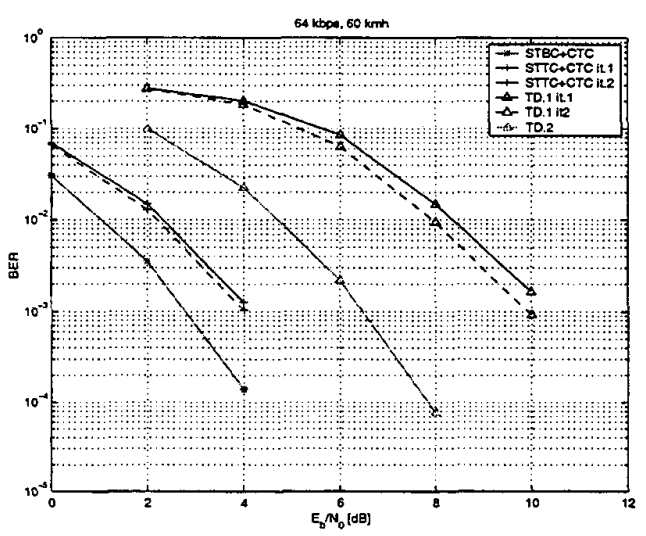

Figure 9: BER for $64 \mathrm{Kbps}$ service at $60 \mathrm{kmh}$

Figure 8 and Figure 9 show the results for a 64 kbps service at 3 and $60 \mathrm{kmh}$, repspectively. We note that TD.2 scheme improves with more than $2 \mathrm{~dB}$ the BER achieved by scheme TD.1. This suggests that is better to perform inter-substream coding, i.e. generate code redundancy through different substreams, rather than independently encode each antenna branch. At $3 \mathrm{kmh}$ there is no improvement by performing more than 2 turbo-decoding iterations. Since the channel remains almost constant during the whole transmission there is no time diversity to be exploited by the interleaver between component codes. At $60 \mathrm{kmh}$ the turbo decoder still improves the BER after 2 iterations. Simulations presented have been run with 4 turbo decoder iterations. We must note that we are assuming perfect channel estimation, however imperfect channel estimation based on the training sequence will decrease the performance. We also observe that no significant iteration gain is achieved by feeding back soft information from the inner to the outer code when using STBC or 4-states STTC neither at $3 \mathrm{kmh}$ nor at $60 \mathrm{kmh}$. Using scheme TD.1 it is possible to obtain an additional (small) iteration gain at $3 \mathrm{kmh}$, but at expenses of high decoding complexity, because feedback information for all (coded and uncoded) bits should be computed. Nevertheless, as shown in Figure 8, this iteration gain is only about $0.5 \mathrm{~dB}$ at same $\mathrm{E}_{\mathrm{b}} / \mathrm{N}_{0}$. At $60 \mathrm{kmh}$, TD. 1 also offers small iteration gain, but in this case only feedback information for uncoded bits was computed.

In Figure 10 and Figure 11 we present performance results for a $384 \mathrm{kbps}$ service at $3 \mathrm{kmh}$ and $60 \mathrm{kmh}$, respectively. The packet length to the encoder is 7680 bits, which is divided in two packets of length 3840 because it is larger than the maximum encode size.

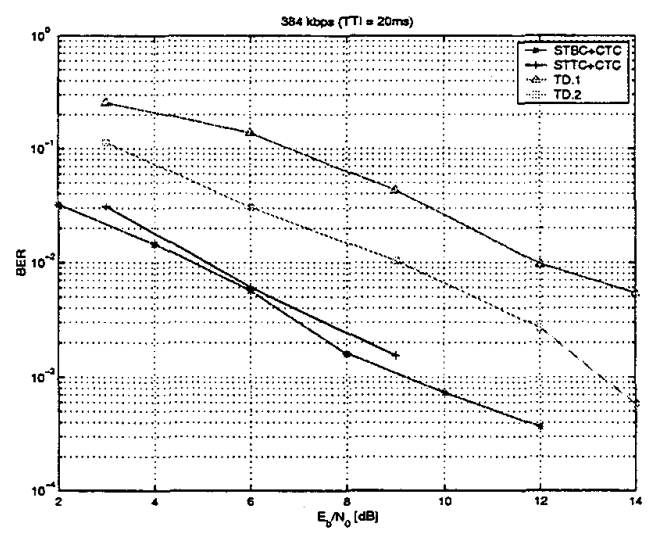

Figure 10: BER for $384 \mathrm{Kbps}$ service at $3 \mathrm{kmh}$

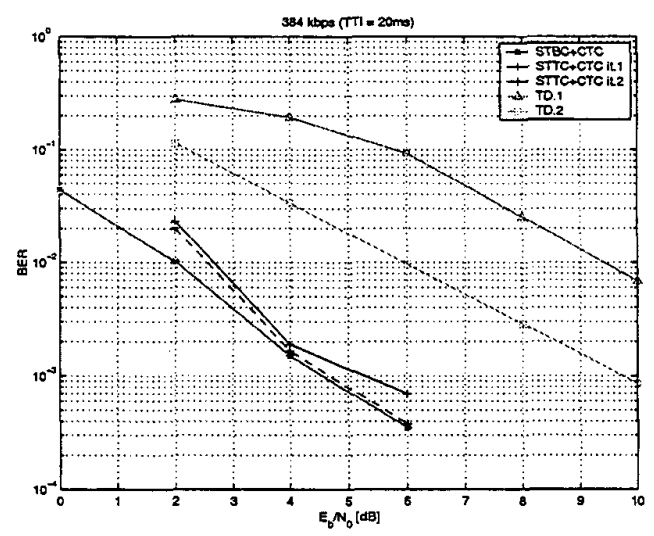

Figure 11: BER for $384 \mathrm{Kbps}$ service at $60 \mathrm{kmh}$ 
Transmit diversity scheme TD. 2 outperforms scheme TD.1. In these simulations only feedback information for uncoded bits was computed and no significant iteration gain was observed.

Finally, Figure 12 shows the performance for a data service of $2 \mathrm{Mbps}$ using STBC or STTC concatenated with a turbo code at $3 \mathrm{kmh}$ and $60 \mathrm{kmh}$. Again, block segmentation is required in order to ensure a correct input packet length to the turbo encoder.

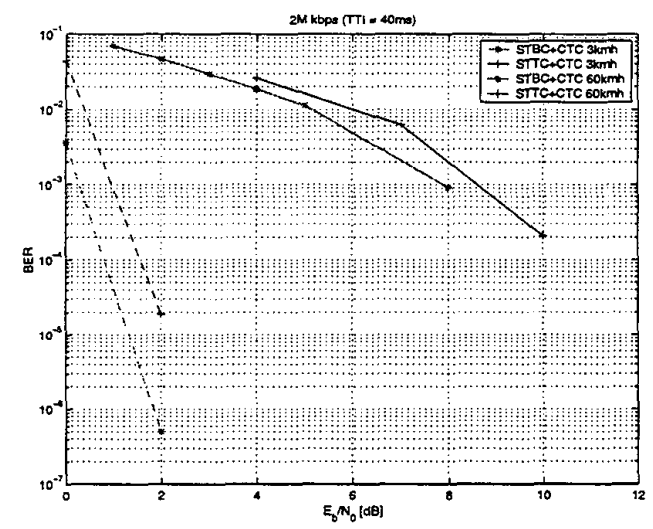

Figure 12: BER for 2 Mbps service.

\section{Conclusions}

In this paper, we presented the performance of different transmit diversity techniques combining space-time codes with an outer channel code using two transmit antennas. In this analysis, STBC outperform 4-states STTC and two different transmit schemes with antenna diversity that do not use space-time coding (TD.1, TD.2). Both STBC and 4-states STTC provide a diversity advantage of 2 but no additional gain can be obtained by using a feedback from the outer code to the inner space-time code.

However, there are many other alternatives to be studied. Long memory STTC or different structures for TD than those presented in this paper based on, for example, multilevel coding could also be considered. Extensions to more than 2 transmit should also be studied. STBC and STTC can easily be extended to higher number of transmit antennas, although STBC with more than two antennas have a rate lower than 1.

Furthermore, channel state estimation should also be considered in the analysis.

\section{References}

[1] V. Tarokh, N. Seshadri, A.R. Calderbank, "Space-Time Codes for High Data Rate Wireless Communication: Performance Criterion and Code Construction", IEEE Trans. IT, vol. 44, no. 2, Mar. 1998, pp.744-765:

[2] V. Tarokh, H. Jafarkhani, A.R. Calderbank, "Space-Time Block Coding for Wireless Communication: Performance Results", IEEE SAC, vol. 17, no. 3, Mar. 1999, pp.451-460.

[3] G.J. Foschini, G.D. Golden, R.A. Valenzuela, P.W. Wolniansky, "Simplified processing for high spectral efficiency wireless communication employing multielement arrays", IEEE Journal on Selected Areas in Communications, vol.17, no.11, p.1841-52, Nov. 1999.

[4] G.D. Golden, G.J. Foschini, R.A. Valenzuela, P.W. Wolniansky, "Detection Algorithm and Initial Laboratory Results using the V-BLAST Space-Time Communication Architecture", Electronics Letters, Vol. 35, No. 1, Jan. 7, 1999, pp. 14-15. Available at http://www.belllabs.com/project/blast.

[5] P.W. Wolniansky, G.J. Foschini, G.D. Golden, R.A. Valenzuela, "V-BLAST: An Architecture for Realizing Very High Data Rates Over the RichScattering Wireless Channel", invited paper, Proc. ISSSE-98, Pisa, Italy, Sept. 29, 1998. Available at http://www.bell-labs.com/project/blast/.

[6] $3^{\text {rd }}$ Generation Partnership Project, Technical Specification Group, Radio Acces Network, Working Group 1 (RANWG1). Pysical channel and mapping of transport channels onto pysical channels (FDD). TS 25.211 v3.1.1. Dec. 1999.

[7] $3^{\text {rd }}$ Generation Partnership Project, Technical Specification Group, Radio Acces Network, Working Group 1 (RANWG1). Multiplexing and channel coding (FDD). TS 25.212 v3.1.1. Dec. 1999.

[8] $3^{\text {rd }}$ Generation Partnership Project, Technical Specification Group, Radio Acces Network, Working Group 1 (RANWG1). Multiplexing and channel coding (TDD). TS 25.222 v3.1.1. Dec. 1999.

[9] C. Berrou, A. Glavieux, P. Thitimajshima, "Near Shannon Limit Error - Correcting Coding and Decoding: Turbo Codes" Proc. IEEE ICC'93, Geneva, Switzerland, May 1993.

[10] Hagenauer, P. Hoeher, "A Viterbi Algorithm with SoftDecisions and its Applications" Proc. IEEE GLOBECOM'89, pp. 1680-1686, November, 1989.

[11] P. Robertson, P. Hoeher, E. Yillebrun, "Optimal and SubOptimal Maximum a Posteriori Algorithms Suitable for Turbo Decoding" ETT Communication Theory, vol. 8, no. 2, pp. 119-125, March-April 1997.

[12] S. Benedetto, D. Divsalar, G. Montorsi, F. Pollara, "Serial Concatenation of Inteleaved Codes: Performance Analysis, Design, and Iterative Decoding", IEEE Trans. IT, vol. 44, no. 3, May 1998, pp.909-925.

[13] G.J.Foschini, "layered Space-Time Architecture for Wireless Communications in a Fading Environment When Using Mulit-Element Antennas", Bell Labs Technichal Journal, Autumn 1996. Available from http://www.lucent.com/minds/techjournal/findex.html.

[14] A. Stefanov, T. Duman, "Turbo Coded Modulation for Wireless Communications with Antenna Diversity", VTC'99, pp.1565-1569. 